\title{
Suicide trends in the elderly during the ongoing COVID-19 Pandemic-A public health urgency
}

\author{
Ashish Sarangi MD, Sozan Fares M.B.B.CH, Noha Eskander M.B.B.CH
}

\begin{abstract}
Background: Older adults have an increased risk for suicide compared to the overall population, and the circumstances surrounding the Coronavirus Disease 2019 (COVID-19) may further increase this risk. COVID-19 pandemic social distancing policies and ethical guidelines for COVID-19 treatment may exacerbate experiences of social isolation, perceived expendability, and exposure to suffering, which are associated with the three main components of the Interpersonal Theory of Suicide: thwarted belongingness, perceived burdensomeness to society, and capability for suicide.

The COVID-19 pandemic places a drain on services and has prompted ethical debates about policies regarding treating younger adults first. These situations may lead older adults to have reduced access to needed medical and psychiatric services and can convey damaging messages of expendability. Furthermore, the potential prolonged stress related to the COVID-19 pandemic may affect neurological, immunological, and health functioning — compounding suicide risk. Potential methods to extend treatment options and reduce social isolation are discussed in this review.

Conclusion: More interaction among all age groups should decrease the mental health distress associated with the COVID-19 pandemic. In particular, older adults need access to healthcare and to family members.
\end{abstract}

Keywords: Geriatric suicide, COVID-19, geriatric depression

\section{INTRODUCTION}

The COVID-19 pandemic continues to affect all aspects of life and older adults are especially vulnerable. Currently, approximately 46 million Americans are over 64 years old. ${ }^{1}$ This population is at substantial risk of psychological consequences from the pandemic, and the COVID-19 pandemic affects the elderly in ways that deserve urgent care and attention. ${ }^{2}$ The World Health Organization (WHO) reports that older adults-particularly those isolated or in cognitive decline-may become more anxious, angry,

Corresponding author: Ashish Sarangi Contact Information: Ashish.Sarangi@ttuhsc.edu DOI: 10.12746/swrccc.v9i40.865 stressed, agitated, and withdrawn while in quarantine. They are also more likely to possess underlying chronic medical conditions like diabetes or respiratory illness-comorbidities known to boost the danger of severe COVID-19 and COVID-19-related death. These trends and risk factors suggest that older adults (both with and without prior psychological symptoms) are in uncharted territory as they experience new or exacerbated psychiatric symptoms. ${ }^{2}$

The pandemic has brought forth pre-existing physical and psychological issues that have increased depression and suicide. ${ }^{3}$ Before COVID-19, about $20 \%$ of individuals age 55 and older experienced either psychological or drug abuse concerns. ${ }^{4}$ While no statistics are yet available to estimate the number of suicides among the elderly due to COVID-19, adults 75 and older have some of the highest suicide 
rates, and although they comprise only $12 \%$ of the U.S. population, older adults account for $18 \%$ of all suicides. ${ }^{5}$ According to the Interpersonal Theory of Suicide, one cause is a concern over being a burden on others or on society. This theory postulates that suicide may be the result of a person's feeling that he or she doesn't belong and that he or she is a burden, combined with an acquired capability for suicide. ${ }^{6}$ Acquired capability for suicide develops over time through repeated exposure to psychological trauma and painful life events.

Strict lockdowns, physical and social distancing, and a sense of being disconnected are factors that could increase rates of depression and anxiety in older adults. ${ }^{7}$ Resource rationing in some healthcare facilities has further compounded the sense of feeling like a burden on the healthcare system in the elderly population. $^{8}$

The mental effects of chronic stress can lead to suicidal thoughts. Seniors may also hear from the media that they are less worthy of care. ${ }^{9}$ An example of this includes news articles portraying the plight of understaffed nursing homes in which tired nurses gave up calling ambulances and paramedics and hospitals at times denying care to the elderly due to their low survival probability. Such portrayals may contribute to suicidal behaviors in older adults by reinforcing negative internalized views on aging as related to a loss of value and productivity as well as feelings of dependency. ${ }^{10}$ Quarantine itself was associated with psychological distress, especially when mandated. ${ }^{11}$ During the SARS outbreak, suicides were reported following enforced quarantine in a Taipei hospital. ${ }^{11}$ Stressors from quarantine associated with poor mental health outcomes in the elderly include frustration and boredom, insufficient information, inadequate supplies, and fear of infection. ${ }^{12}$

The objective of this literature review is to explore how COVID-19 related isolation and lockdowns contributed to the increase in the risk of suicide in the elderly population. This research review presents solutions to this isolation and discusses new challenges faced by older people during the COVID-19 pandemic.

\section{METHODS}

Relevant literature was searched for on Pub Med using the keywords "elderly" and "COVID-19." Included articles had publication dates within the last 29 years, were in English, were about humans, and were systematic reviews, narrative reviews, clinical trials, randomized clinical trials, or meta-analyses. Articles about individuals less than 65-years-old were excluded.

Thirty-two articles met the selection criteria. A systematic review in this study was not performed, and no quality assessment of the selected research studies was done.

\section{Discussion}

The fact that social connections have a lead role in suicide prevention has been supported by leading psychological theories. Individuals struggling with suicidal ideation often self-isolate and lose connections to people and the outside world, increasing suicide risk. ${ }^{13}$ From a suicide prevention perspective, it is concerning that the most critical public health strategy for the COVID-19 crisis is social distancing, causing family and friends to remain at a distance from individuals who are hospitalized in inpatient psychiatric care units.

Thwarted belongingness includes loneliness and therefore the absence of reciprocal care. People have an innate need to belong and to be a part of families, social groups, and communities. ${ }^{13}$ As people age, they often lose some existing societal supports, e.g., retiring from work, loss of friends to death, and illness. Nonetheless, socioemotional selectivity theory suggests that relationship satisfaction and social wellbeing increase in later life. ${ }^{14}$ The reasons for this include motivational shifts in the elderly, which leads to greater selectivity in choosing friends and allocation of greater resources toward a smaller social circle.

One study examining COVID-19 pandemic-related increase in anxiety found that age was inversely associated with isolation anxiety. ${ }^{15}$ However, on average, older adults tend to have smaller social networks, ${ }^{16}$ and disruption of those smaller networks through 
social distancing practices may contribute to increased social isolation. More broadly, because increased age is related to higher rates of chronic health conditions, older adults could also be more conscientiously engaged in social distancing practices, avoiding people, situations, and daily interactions that they would normally enjoy. Van Orden et al. recently stated that the disruption of social networks may cause greater isolation among older adults with potentially serious consequences to health. Social connectedness is vital to physical and mental health across a person's lifetime. ${ }^{17}$

Olaya et al. reported that among adults older than 50 , social isolation is related to an increased risk for multiple chronic diseases. The absence of positive social relationships and loneliness among older adults are significant risk factors for broad-based morbidity, mortality, ${ }^{18}$ and suicide. ${ }^{19}$ Shrira et al. found that subjective age, which is how young or old one perceives himself/herself to be, may strengthen the association between loneliness and psychiatric symptoms during the COVID-19 pandemic. $^{20}$ Thus, it is important to contemplate how social isolation associated with social distancing policies may affect the health and psychological functioning of older people and could exacerbate existing risks for suicide.

Perceived burdensomeness is a component of the interpersonal theory of suicide. It is the belief that one is a burden on others or society. Some older adults are at a higher risk of experiencing perceived burdensomeness due to higher rates of chronic health conditions. ${ }^{21}$ For example, Cukrowicz et al. found, in two samples of older adults (mean age $=74.1 \pm 7.5$ years and mean age $=70.9 \pm 7.6$ years), that poor health was associated with perceived burdensomeness and suicide ideation. ${ }^{21,22}$

The causes of suicide in China during the severe acute respiratory syndrome epidemic among individuals 65 years and older were related to concerns of being infected and becoming a burden to their families. ${ }^{23}$ Meta-analyses conducted by $\mathrm{Chu}$ et al. revealed that the connection between perceived burdensomeness and suicidality was especially robust; however, the association was not moderated by age.

During the COVID-19 pandemic, it has been well publicized that the severity of COVID-19 often depends on chronic health conditions with mortality rates increasing with increasing ages (odds ratio: 1.1, 95\% confidence interval: $1.03-1.17, p=.0043){ }^{24}$ Thus, for older adults with pre-existing conditions, fear of contracting COVID-19 and likely requiring a higher level of care potentially increases concerns over being a burden.

The interpersonal theory of suicide views exposure to pain, injury, and death as a crucial driver of the capability to die by suicide. Frequent and intense exposure to such bodily related themes lessens the fear of these very topics-thus increasing capability. Indeed, the topics of physical suffering and COVID-19related death are reported in the news daily; this media coverage has been relentless. Worldwide there are millions of COVID-19 infections; approximately $7 \%$ of those have died, with death rates increasing by age group (WHO Coronavirus [COVID-19] Dashboard, https://covid19.who.int/).

Considering typical social networks, it is likely that many people personally know someone who has contracted COVID-19; others are still exposed to people affected by COVID-19, e.g., via news outlets, as themes of morbidity and mortality have surged. However, research showing an association between such media exposure and increased capability for suicide is lacking.

Certain subpopulations have elevated suicide rates due to exposure over time to long-existing, high levels of capability, e.g., via current or past occupational exposure; health care professionals and first responders are also at particular risk. ${ }^{25}$ Indeed, 20\% of current first responders are older than $55 .^{26}$ Thus, the COVID-19 pandemic may increase population levels of suicide capability due to dramatically increased exposure to suffering and death.

There are several ways in which the COVID-19 pandemic will have an impact on suicide in older adults, not limited to increasing the prevalence of known risk factors for suicide and infection control measures that increase isolation and vulnerability. As countries continue to grapple with economic, political, and natural disaster challenges during the pandemic, suicide risk in the elderly needs to be carefully monitored. There are common elements to suicide prevention in older adults including disseminating accurate information, promoting self-help and positive coping, 
reducing isolation through technology, and developing telehealth.

Physician education in depression recognition and treatment and restricting access to lethal methods can reduce suicide rates. Other interventions need more evidence of efficacy. Determining which components of suicide prevention programs are effective in reducing rates of suicide and suicide attempt is essential to maximize the use of limited resources.

\section{Population approaches (PRIMARY PREVENTION)}

Various organizations have issued advice for coping with anxiety and stress during the COVID-19 pandemic, which may reduce suicide risk. The Australian Psychological Society (APS) has issued tips for older adults to stay mentally healthy. ${ }^{27}$ Information is important to reduce the risk of psychological distress, including providing older people with a clear rationale for why self isolation is important, general education about the virus to reduce stigmatization, and emphasizing the altruistic decision to stay home. ${ }^{28}$

Conveying such information via television may be an effective approach that reaches many older people. The APS also recommends seeking information from reliable sources and in moderation, keeping concerns in perspective, and using existing healthy coping skills. There are suggestions for coping with social distancing by using videoconferencing, text messaging, phone calls, and e-mails with friends and family instead of face-to-face meetings.

A sense of belonging, connectedness, and social support can be derived through online technologies, addressing key suicide risk factors. For some older people, this will involve learning new skills; Australians aged $65+$, for example, are the most digitally excluded population group, least able to use digital technology for social connections and to access information and services. $^{28}$

\section{ContinUity of ACCESS TO MENTAL HEALTH CARE (SECONDARY AND TERTIARY PREVENTION)}

Community mental health services should review their patient lists and screen for or otherwise identify older clients who are especially vulnerable to mental illness and suicide, e.g., those who live alone, were already socially isolated, have chronic medical comorbidities or functional disability, are currently unwell, or are at risk of relapse, and institute regular welfare checks and enhanced follow-up. ${ }^{29}$ Patients receiving depot antipsychotics or requiring regular medication monitoring, e.g., blood tests for lithium or clozapine, may need additional support from mental health services to ensure continued access to treatment, especially for those whose general practitioners have reduced hours or closed and no longer provide this care. People who contract COVID-19 and have suicide risk factors should be actively followed.

Telehealth has become important during the COVID-19 pandemic and may improve the access of older people internationally to mental health care now, but also later if embedded in mainstream health care. This involves switching from a hospital and clinicbased model of mental health care to telephone- and internet-based services and increasing public awareness about where and how to access the off-site services.

There are online psychotherapy courses available for those suffering from anxiety and depressive illnesses (see https://thiswayup.org.au/) and for loneliness, although specific telehealth treatments for suicidal ideation are less developed. Grief counseling for those bereaved during the pandemic could also be delivered online. Older people may require additional support and education from families, friends, and healthcare professionals to access these services.

Informal and professional services have a role in reducing social isolation-a factor increasing suicide risk-in older people during the COVID-19 pandemic. Grassroots initiatives to reduce loneliness have emerged such as \#TheKindnessPandemic, developed by Celebrate Ageing, to promote acts of intergenerational kindness. Children can be encouraged to keep in contact with their older parents and grandparents to reduce fear and loneliness. ${ }^{30}$ For those without relatives, support services could be provided by community workers.

Online technologies can also be used to promote a sense of belonging and provide social support for 
older people. ${ }^{31}$ Formal services, such as Tele-Help/ Tele-Check in Italy, telephone support, and monitoring, have demonstrated benefits in suicide prevention for older people. ${ }^{32}$ This model of proactive connection of older adults with health services via telephone could be used to provide home assistance to older people at risk of suicide due to social isolation, and/or psychological or physical illness. Variations of this approach could be delivered by family and friends, charities, voluntary organizations, or healthcare professionals. Telephone crisis line services have played a role in suicide prevention and crisis support in the community providing an inexpensive, convenient, and anonymous means of seeking support with the potential to reach a large proportion of community-dwelling older people. ${ }^{32,33}$

Although quarantine is necessary to reduce the spread of COVID-19, measures can be taken to reduce the factors associated with poor mental health. This could include a public health campaign explaining why quarantine is important, minimizing the total duration of the quarantine period, ensuring older people access to sufficient food, household essentials, and medicine (through welfare strategies such as those implemented in China), and promoting suggestions for home-based activities to limit boredom.

\section{Conclusions}

There may be unintended adverse consequences of policies to prevent contagion for older people. Advice regarding exercise and movement outside the home varies among countries and age groups, but social distancing may lead to more physical deconditioning, greater pain, and ultimately greater disability for older people. Each of these negative sequelae is also a risk factor for suicide in older people. Exercise is also an effective treatment for depression, ${ }^{34}$ and, if no longer available as a coping strategy for older adults, could potentially increase suicide risk. More interaction among all age groups should decrease the mental distress associated with the COVID-19 pandemic. In particular, older adults need access to health care and to family members. Finally, the public should acknowledge the optimistic benefit of "pulling together" as a society and the many valuable ways older adults can contribute during this crisis.
Article citation: Sarangi A, Fares S, Eskander N. Suicide trends in the elderly during the ongoing COVID-19 Pandemic-A public health urgency. The Southwest Respiratory and Critical Care Chronicles 2021;9(40):31-36 From: Department of Psychiatry (AS), Texas Tech University Health Sciences Center, Lubbock, Texas; Hawler Medical University (SF), Iraq; Ain Shams Faculty of Medicine (NE), Egypt

Submitted: $4 / 25 / 2021$

Accepted: $7 / 7 / 2021$

Reviewer: John Culberson MD

Conflicts of interest: none

This work is licensed under a Creative Commons Attribution-ShareAlike 4.0 International License.

\section{REFERENCES}

1. Sarangi A, Nelson J. A descriptive Study Evaluating the Impact of COVID-19 on delivery of care and mental health of geriatric nursing home staff. Am J Geriatric Psychiatry 2021; 29(4),S121-S122.

2. Chan SS, Leung VP, Tsoh J, et al. Outcomes of a two-tiered multifaceted elderly suicide prevention program in a Hong Kong Chinese community. Am J Geriatric Psychiatry 2011; 19(2):185-196.

3. American Foundation for Suicide Prevention. 2021 [cited 2021 Jul 3]; Available from: https://afsp.org/

4. Advancing independence, integration, and inclusion throughout life [Internet]. ACL Administration for Community Living. 2019 [cited 2021 Jul 3]; Available from: https://acl.gov/

5. Advanced Solutions International I. American Association for Marriage and Family Therapy. 2021 [cited 2021 Jul 3]; Available from: https://www.aamft.org/

6. Van Orden KA, Witte TK, Cukrowicz KC, et al. The interpersonal theory of suicide. Psychol Rev 2010;117(2):575-600.

7. Santini ZI, Jose PE, York Cornwell E, et al. Social disconnectedness, perceived isolation, and symptoms of depression and anxiety among older Americans (NSHAP): a longitudinal mediation analysis. Lancet Public Health 2020;5(1):e62-e70.

8. Rosenbaum L. Facing Covid-19 in Italy-ethics, logistics, and therapeutics on the epidemic's front line. N Engl J Med 2020;382(20):1873-1875.

9. News: Op-Ed: As hospitals become overwhelmed ... (The Los Angeles Times)-Behind the headlines-NLM [Internet]. National Center for Biotechnology Information. 2020 [cited $2021 \mathrm{Jul}$ 3]; Available from: https://www.ncbi.nlm.nih.gov/ search/research-news/9089/ 
10. Crocker L, Clare L, Evans K. Giving up or finding a solution? The experience of attempted suicide in later life. Aging Mental Health 2006;10(6):638-647.

11. Brooks SK, Webster RK, Smith LE, et al. The psychological impact of quarantine and how to reduce it: rapid review of the evidence. Lancet 2020;395(10227):912-920.

12. Barbisch D, Koenig KL, Shih FY. Is there a case for quarantine? perspectives from SARS to Ebola. Disaster Med Public Health Prep 2015;9(5):547-553.

13. Hawkley LC, Capitanio JP. Perceived social isolation, evolutionary fitness and health outcomes: a lifespan approach. Philos Trans R Soc Lond B Biol Sci. 2015;370(1669):20140114. doi:10.1098/rstb.2014.0114.

14. Carstensen LL. Social and emotional patterns in adulthood: Support for socioemotional selectivity theory. Psychology and Aging 1992;7(3):331-338.

15. Bareket-Bojmel L, Shahar G, Margalit M. COVID-19related economic anxiety is as high as health anxiety: findings from the USA, the UK, and Israel [published online ahead of print, 2020 May 29]. Int J Cogn Ther 2020;1-9. doi:10.1007/ s41811-020-00078-3.

16. Charles ST, Carstensen LL. Social and emotional aging. Annual Review Psychology 2010; 61:383-409.

17. Holt-Lunstad J. Why social relationships are important for physical health: a systems approach to understanding and modifying risk and protection. Annu Rev Psychol 2018;69: 437-458.

18. Cacioppo JT, Cacioppo S. Social relationships and health: the toxic effects of perceived social isolation. Soc Personal Psychol Compass 2014;8(2):58-72.

19. Fässberg MM, van Orden KA, Duberstein $P$, et al. A systematic review of social factors and suicidal behavior in older adulthood. Int J Environ Res Public Health 2012;9(3):722-745.

20. Shrira A, Hoffman Y, Bodner E, et al. COVID-19 related loneliness and psychiatric symptoms among older adults: The buffering role of subjective age. American J Geriatric Psychiatry 2020. doi: 10.1016/j.jagp.2020.05.018

21. Gavrilov LA, Gavrilova NS. Is aging a disease? Biodemographers' point of view. Advances in Gerontology 2018;8(2): $123-124$.

22. Cukrowicz KC, Cheavens JS, Van Orden KA, et al. Perceived burdensomeness and suicide ideation in older adults. Psychology Aging 2011;26(2), 331-338.
23. Yip PS, Cheung YT, Chau PH, et al. The impact of epidemic outbreak: the case of severe acute respiratory syndrome (SARS) and suicide among older adults in Hong Kong. Crisis 2010;31(2):86-92.

24. Zhou F, Yu T, Du R., et al. Clinical course and risk factors for mortality of adult inpatients with COVID-19 in Wuhan, China: a retrospective cohort study. Lancet (London, England) 2020;395(10229):1054-1062.

25. Stanley I H, Hom M A, Joiner T E. A systematic review of suicidal thoughts and behaviors among police officers, firefighters, EMTs, and paramedics. Clinical Psychology Review 2016;44,25-44.

26. Schafer K, Sutter R, Gibbon S. Characteristics of individuals and employment among first responders. US Department of Labor 2015. DOI: 10.13140/RG.2.1.2438.0640

27. Australian Psychological Society. Psychology and Ageing Interest Group Committee (2020). Coronavirus (COVID-19) Anxiety and Staying Mentally Healthy for Older Adults. Victoria: Australian Psychological Society. psychology.org.au

28. Thomas J, Barrakat J, Wilson C, et al. Measuring Australia's digital divide: The Australian digital inclusion index 2017. Melbourne: RMIT University. DOI: 10.4225/50/ $596473 \mathrm{db} 69505$

29. Reger MA, Stanley IH, Joiner TE. Suicide mortality and Coronavirus disease 2019-a perfect storm? JAMA Psychiatry 2020 Nov 1;77(11):1093-1094.

30. Käll A, Jägholm $S$, Hesser $H$, et al. Internet-based cognitive behavior therapy for loneliness: a pilot randomized controlled trial. Behav Ther 2020;51(1):54-68.

31. Newman MG, Zainal NH. The value of maintaining social connections for mental health in older people. Lancet Public Health 2020 Jan;5(1):e12-e13.

32. De Leo D, Dello Buono M, Dwyer J. Suicide among the elderly: the long-term impact of a telephone support and assessment intervention in northern Italy. Br J Psychiatry 2002 Sep;181:226-9.

33. Krysinska KE, De Leo D. Telecommunication and suicide prevention: hopes and challenges for the new century. Omega (Westport) 2007;55(3):237-253.

34. Fässberg MM, Cheung G, Canetto SS, et al. A systematic review of physical illness, functional disability, and suicidal behaviour among older adults. Aging Mental Health 2016; 20(2):166-194. 